\title{
Comprehensive characterization of cardiac morphology and function in adult patients with phenylketonuria using CMR
}

\author{
Jan-Hendrik Hassel', Nikolaus Tilling², Lenka Bosanska², Bernhard Schnackenburg ${ }^{3}$, Daniel Messroghli', \\ Alexander Berger ${ }^{1}$, Rolf Gebker ${ }^{1}$, Christopher Schneeweis ${ }^{1}$, Eckart Fleck', Ursula Plöckinger ${ }^{2}$, Sebastian Kelle ${ }^{1 *}$
}

From 17th Annual SCMR Scientific Sessions

New Orleans, LA, USA. 16-19 January 2014

\section{Background}

Phenylketonuria (PKU) is one of the most common inherited metabolic disorders. The molecular pathway of neurological damage is not yet sufficiently understood. To date, there is a lack information about cardiac involvement related to the disease. This study aims to characterize cardiac morphology and function in adult patients with PKU using cardiovascular magnetic resonance (CMR).

\section{Methods}

28 patients with PKU (age $30 \pm 9$ years/mean \pm SD) underwent a comprehensive CMR protocol at a $1.5 \mathrm{~T}$ CMR scanner (Philips, Achieva) including assessment of left ventricular (LV) volume and mass. In addition, T1 measurements pre- and post-administration of gadolinium for evaluation of extra corpuscular volume (ECV) and tagging for quantitative analysis of left ventricular circumferential strain (Ecc) were performed. 8 healthy age-matched volunteers underwent a similar protocol and served as controls for the ECV values. LV parameters and Ecc were compared to reference values from previous studies with similar data setup [1-3].

\section{Results}

CMR exams were successfully performed in all patients. As shown in Figure $1 \mathrm{LV}$ mass index was reduced to the lower $95 \%$ confidence interval of the reference values $[4,5]$ in each subgroup. ECV showed no significant difference between PKU patients $(0.27 \pm 0.03 /$ mean \pm SD) and the control group $(0.28 \pm 0.02 /$ mean $\pm \mathrm{SD})$ (Figure 2) $\mathrm{p}=0.15$. PKU patients had higher Ecc values $(=-0.22 \%)$ compared to reference values $(\mathrm{Ecc}=-0.20 \%)$ with similar segmental patterns.

\section{Conclusions}

The results of this study indicate that in PKU patients compared to healthy controls LV mass indexed to BSA is reduced to the reference values; we found increased

\begin{tabular}{|c|c|c|c|c|c|c|c|c|c|}
\hline $\begin{array}{l}\text { Tbl.1 } \\
\text { F 20-29 } \\
\text { F } 30-39 \\
\text { F 40-49 }\end{array}$ & $\begin{array}{l}6 \\
5 \\
3\end{array}$ & $\begin{array}{c}\begin{array}{c}\text { EDVI LV } \\
{\left[\mathbf{m} \mid \mathbf{m}^{2}\right]}\end{array} \\
\mathbf{8 8 . 5 3} \pm 23,32 \\
\mathbf{7 4 . 9 8} \pm 11,19 \\
\mathbf{9 0 . 3 1} \pm 2,43\end{array}$ & $\begin{array}{c}\begin{array}{c}\text { Reference } \\
\text { Range }\end{array} \\
82(65-99) \\
79(62-96) \\
76(59-93)\end{array}$ & $\begin{array}{c}\begin{array}{c}\text { ESVI LV } \\
{\left[\mathbf{m} \mid \mathbf{m}^{2}\right]}\end{array} \\
\mathbf{3 5 . 7 4} \pm 11,50 \\
\mathbf{3 3 . 3 9} \pm 4,43 \\
\mathbf{3 4 . 6 3} \pm 6,29\end{array}$ & $\begin{array}{c}\begin{array}{c}\text { Reference } \\
\text { Range }\end{array} \\
28(19-37) \\
27(17-36) \\
25(16-34)\end{array}$ & $\begin{array}{c}\text { SVI LV } \\
{\left[\mathbf{m} \mid \mathbf{m}^{2}\right]} \\
\mathbf{5 2 . 7 9} \pm 13,40 \\
\mathbf{4 1 . 5 9} \pm 7,59 \\
\mathbf{5 5 . 6 8} \pm 3,86\end{array}$ & $\begin{array}{c}\begin{array}{c}\text { Reference } \\
\text { Range }\end{array} \\
54(42-66) \\
53(40-65) \\
51(39-63)\end{array}$ & $\begin{array}{c}\text { Mass Index } \\
\text { LV }\left[\mathrm{g} / \mathrm{m}^{2}\right]\end{array}$ & $\begin{array}{c}\begin{array}{c}\text { Reference } \\
\text { Range }\end{array} \\
62(47-77) \\
62(47-77) \\
63(48-77)\end{array}$ \\
\hline $\begin{array}{l}\text { M 20-29 } \\
\text { M 30-39 } \\
\text { M 40-49 }\end{array}$ & $\begin{array}{l}7 \\
5 \\
2\end{array}$ & $\begin{array}{c}\mathbf{8 8 . 6 9} \pm 13,40 \\
\mathbf{8 7 . 9 0} \pm 17,62 \\
\mathbf{7 5 . 9 0} \pm 7,45\end{array}$ & $\begin{array}{c}86(68-103) \\
83(66-101) \\
81(64-99)\end{array}$ & $\begin{array}{c}\mathbf{4 2 . 2 8} \pm 5,53 \\
\mathbf{4 0 . 4 0} \pm 10,64 \\
\mathbf{3 8 . 4 4} \pm 4,53\end{array}$ & $\begin{array}{l}30(19-41) \\
29(18-39) \\
27(17-38)\end{array}$ & $\begin{array}{l}\mathbf{4 6 . 4 1} \pm 8,91 \\
\mathbf{4 7 . 5 1} \pm 8,06 \\
\mathbf{3 7 . 4 5} \pm 2,92\end{array}$ & $\begin{array}{l}56(44-68) \\
55(43-67) \\
54(42-66)\end{array}$ & $\begin{array}{l}\mathbf{5 0 . 0 5} \pm 11,27 \\
\mathbf{4 1 . 4 7} \pm 10,35 \\
\mathbf{3 3 . 0 8} \pm 11,05\end{array}$ & $\begin{array}{l}76(59-93) \\
75(59-92) \\
75(58-91)\end{array}$ \\
\hline $\begin{array}{l}F=\text { femal } \\
\text { e } \\
M=\text { male }\end{array}$ & & mean $\pm S D$ & $\begin{array}{l}\text { mean (95\% } \\
\text { confidence } \\
\text { interval) }\end{array}$ & mean $\pm S D$ & $\begin{array}{l}\text { mean ( } 95 \% \\
\text { confidence } \\
\text { interval) }\end{array}$ & mean $\pm S D$ & $\begin{array}{c}\text { mean (95\% } \\
\text { confidence } \\
\text { interval) }\end{array}$ & mean $\pm S D$ & $\begin{array}{c}\text { mean }(95 \% \\
\text { confidence } \\
\text { interval) }\end{array}$ \\
\hline
\end{tabular}

${ }^{1}$ Cardiology, German Heart Institute Berlin, Berlin, Germany

Full list of author information is available at the end of the article 


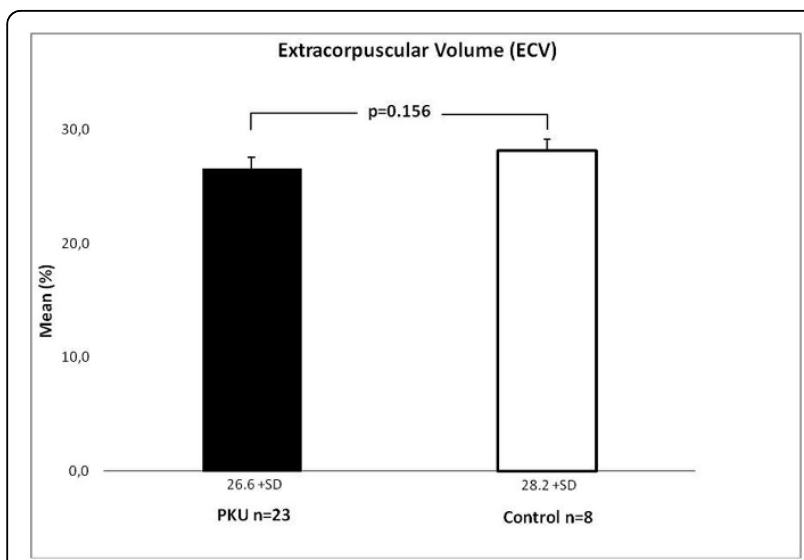

Figure $2 \mathrm{ECV}$ in patients with phenylketonuria and healthy controls.

average Ecc parameters and normal ECV values. Further investigations in larger patient groups and older PKU patients are necessary for evaluation of cardiac involvement of the disease over time and its consequences.

\section{Funding}

None.

\section{Authors' details}

${ }^{1}$ Cardiology, German Heart Institute Berlin, Berlin, Germany. ${ }^{2}$ Interdisziplinäres Stoffwechsel-Centrum, Charité-Universitätsmedizin Berlin, Campus VirchowKlinikum, Berlin, Germany. ${ }^{3}$ Philips Healthcare Systems, Hamburg, Germany.

Published: 16 January 2014

\section{References}

1. Kuijer, et al: Three-dimensional myocardial strains at end-systole and during diastole in the left ventricle of normal humans. J Cardiovasc Magn Reson 2002, 4(3):341-351.

2. Moore, et al: Three-dimensional systolic strain patterns in the normal human left ventricle: characterization with tagged MR imaging In Radiology 2000, 214(2):453-466.

3. Young, et al: Three-dimensional left ventricular deformation in hypertrophic cardiomyopathy. In Circulation 1994, 90(2):854-867.

4. Maceira, et al: Normalized left ventricular systolic and diastolic function by steady state free precession cardiovascular magnetic resonance. In J Cardiovasc Magn Reson 2006, 8(3):417-426.

5. Maceira, et al: Reference right ventricular systolic and diastolic function normalized to age, gender and body surface area from steady-state free precession cardiovascular magnetic resonance. In Eur Heart J 2006, 27(23):2879-2888

doi:10.1186/1532-429X-16-S1-P246

Cite this article as: Hassel et al:: Comprehensive characterization of cardiac morphology and function in adult patients with phenylketonuria using CMR. Journal of Cardiovascular Magnetic Resonance 2014 16(Suppl 1):P246.

\section{Submit your next manuscript to BioMed Central and take full advantage of:}

- Convenient online submission

- Thorough peer review

- No space constraints or color figure charges

- Immediate publication on acceptance

- Inclusion in PubMed, CAS, Scopus and Google Scholar

- Research which is freely available for redistribution

Submit your manuscript at www.biomedcentral.com/submit
C Biomed Central 